\title{
Study of Secondary Field Waves at Scattering of Nonlinearly Interacting Acoustic Waves by an Elongated Spheroid
}

\author{
Iftikhar B. Abbasov \\ Taganrog Technological Institute of the Southern Federal University, Nekrasovskyi 44, Taganrog, 347928, Russia
}

(Received 25 October 2013; revised 1 April 2008; accepted 6 October 2014)

\begin{abstract}
The study and three-dimensional simulation of the field of the second harmonic wave at the scattering of nonlinearly interacting acoustic waves by an elongated spheroid are carried out in this work. The problem is presented in the elongated spheroidal coordinate system, and the foci of the spheroid coincide with foci of the spheroidal coordinate system. The description of the occurring wave processes is presented on the basis of the obtained relation for acoustic pressure of the second harmonic wave. The scattering diagrams for the acoustic pressure field of the second harmonic wave are presented, and three-dimensional models of a scattering diagram are created.
\end{abstract}

\section{INTRODUCTION}

The problem of acoustic waves scattering by elongated spheroids was formulated for the first time about half a century ago. ${ }^{1-3}$ Some of this research considered the problem of the sound scattering by an elongated spheroid with different boundary conditions. ${ }^{1}$ Other research considered the problem of the sound scattering by a rigid spheroid in the long-wave approximation. $^{2}$ And other work is devoted to the problem of sound waves scattering by an elongated spheroid. ${ }^{3}$ The angular characteristics of the acoustic waves scattering by a soft and rigid elongated spheroid were presented.

The process of the plane acoustic waves scattering by thin, acoustically rigid and resilient bodies of revolution was considered in some of the research used in this study. ${ }^{4,5}$ In other works, the problem of the plane acoustic wave scattering by spheroidal shells was investigated. 6,7 These works study the surface waves directly on scatterers, and the frequency dependence of the backscattering amplitude in the far field is presented. With the exception of the works on the linear scattering by spheroids, there are several papers devoted to nonlinear acoustic spectroscopy. Some studies used here consider the problem of nonlinear acoustic diagnostics of defects in materials and structures. ${ }^{8}$ Other research presented here is devoted to solving the problem of the nonlinear acoustic spectroscopy of defects in geomaterials. ${ }^{9}$

However, the problem of interacting nonlinear acoustic waves scattered by an elongated spheroid has not been examined elsewhere. This problem becomes essential when an acoustic parametric antenna is used for remote diagnostics of a water medium, as well as in the medical tomography field.

In the present work, the study and simulation of the secondary field of the second harmonic wave is carried out. The study of the secondary field of the difference-frequency wave for nonlinearly interacting plane acoustic waves scattered by a rigid elongated spheroid was also performed. ${ }^{10}$ However, the scattering process for the high-frequency second harmonic wave has a purely geometrical character $\left(k_{2 \omega} h_{0} \gg 1\right)$. But the difference-frequency wave covers the Rayleigh- $\left(k_{-} h_{0} \ll 1\right)$ and resonance- $\left(k_{-} h_{0} \approx 1\right)$ scattering regions. A multifrequency analysis will lead to an increase in informativeness of the scattered signal.

\section{STATEMENT OF PROBLEM}

The problem of wave diffraction by elongated bodies is often described in ellipsoidal coordinates. These coordinates are used in the study of radiation and the scattering of acoustic waves by ellipsoids, or cigar-shaped bodies, and when studying the diffraction by circular apertures. ${ }^{11}$

The elongated spheroidal coordinate system, $\xi, \eta$, and $\varphi$, is used for the study of diffraction by cigar-shaped bodies. The foci of the spheroid coincide with foci of the spheroidal coordinate system. The spheroid is formed by rotating the ellipse $\xi_{0}$ around its major axis, which coincides with the $x$-axis in the Cartesian coordinate system. The geometry of the problem is presented in Fig. 1. The $\xi=$ const spheroids and $\eta=$ const two-sheeted hyperboloids are the coordinate surfaces in this case.

Elongated spheroidal coordinates are related to Cartesian coordinates by the following equations: ${ }^{12}$

$$
\begin{gathered}
x=h_{0} \xi \eta, \quad y=h_{0} \sqrt{\left(\xi^{2}-1\right)\left(1-\eta^{2}\right)} \cos \varphi \\
z=h_{0} \sqrt{\left(\xi^{2}-1\right)\left(1-\eta^{2}\right)} \sin \varphi
\end{gathered}
$$

where $h_{0}=d / 2$ and $d$ is the interfocal distance. Spheroidal coordinates $\xi, \eta$, and $\varphi$ vary within the limits $1 \leq \xi<\infty$; $-1 \leq \eta \leq 1$; and $0 \leq \varphi \leq 2 \pi$.

The perfect elongated spheroid is placed into a homogeneous medium. The spheroid's surface is characterized by the radial coordinate $\xi_{0}$. In our case, the spheroid is supposed to be acoustically rigid. Consequently, the Neumann boundary 